\title{
Verhandlungen der
}

\section{Deutschen Gesellschaft für Innere Medizin}

Herausgegeben von dem ständigen Schriftführer K. Miehlke

92. Kongreß

Gehalten zu Wiesbaden vom 6. bis 10. April 1986

Mit 298 Abbildungen und 248 Tabellen

Referate zu folgenden Hauptthemen: Der leise Beginn chronischer Krankheiten: Beiträge zu ihrer Frühsymptomatologie und zur Nosologie der Frühstadien - Grundlagenforschung als Voraussetzung medizinischer Fortschritte - Gemeinsame Gebiete von Innerer Medizin und Chirurgie - Ernährung und Krankheit: Aktuelle Probleme und ihre Lösung

Rundtischgespräche zu folgenden Themen: Das koronare Risiko - AIDS: Entwicklungen seit 1984 - Therapeutische Grenzgebiete von Innerer Medizin und Chirurgie an Beispielen aus der Gastroenterologie

Symposien zu folgenden Themen: Angeborene Störungen des Lipidstoffwechsels: Pathogenese und Therapie - Chance und Risiko: Alternativen zwischen palliativer und kurativer Therapie - Gicht und andere Störungen des Purin- und Pyrimidinstoffwechsels Der Typ II Diabetes - Polyenfettsäuren und Eicosanoide: Ihre klinische Bedeutung heute - Wahl der Nahrungsproteine: Grundlagen und Diätetik

\section{J. F. Bergmann Verlag München}


Professor Dr. Klaus Miehlke

Humboldtstr. 14

D-6200 Wiesbaden

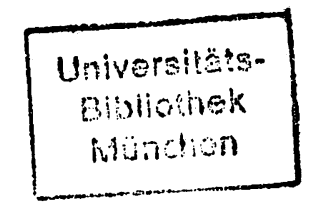

ISBN 3-8070-0360-6 J. F. Bergmann Verlag München

ISBN 0-387-00360-6 Springer-Verlag New York Berlin Heidelberg London Paris Tokyo

CIP-Kurztitelaufnahme der Deutschen Bibliothek:

Deutsche Gesellschaft für Innere Medizin:

Verhandlungen der Deutschen Gesellschaft für

Innere Medizin : . . Kongress. - München : J. F. Bergmann

ISSN 0070-4067

92. Gehalten zu Wiesbaden vom 6. bis 10. April 1986. - 1986.

ISBN 3-8070-0360-6 (Bergmann)

ISBN 0-387-00360-6 (Springer, New York ...)

Das Werk ist urheberrechtlich geschützt. Die dadurch begründeten Rechte, insbesondere die der Übersetzung, des Nachdruckes, der Entnahme von Abbildungen, der Funksendung, der Wiedergabe auf photomechanischem oder ähnlichem Wege und der Speicherung in Datenverarbeitungsanlagen bleiben, auch bei nur auszugsweiser Verwertung, vorbehalten.

Die Vergütungsansprüche des $\S 54$, Abs. 2 UrhG werden durch die „Verwertungsgesellschaft Wort", München, wahrgenommen.

(1) J. F. Bergmann Verlag, München 1986

Printed in Germany

Die Wiedergabe von Gebrauchsnamen, Handelsnamen, Warenbezeichnungen usw. in diesem Werk berechtigt auch ohne besondere Kennzeichnung nicht zu der Annahme, daß solche Namen im Sinne der Warenzeichen- und Markenschutz-Gesetzgebung als frei zu betrachten wären und daher von jedermann benutzt werden dürften.

Produkthaftung: Für Angaben über Dosierungsanweisungen und Applikationsformen kann vom Verlag keine Gewähr übernommen werden. Derartige Angaben müssen vom jeweiligen Anwender im Einzelfall anhand anderer Literaturstellen auf ihre Richtigkeit überprüft werden.

Satz, Druck und Einband: Graphischer Betrieb Konrad Triltsch GmbH, 8700 Würzburg

Verantwortlich für den Anzeigenteil:

E. Lückermann, H. Hüttig, Kurfürstendamm 237, D- 1000 Berlin 15

2382/3321-543210 


\section{Inhaltsverzeichnis}

Vorsitzender 1986-1987 . . . . . . . . . . . . . . . . . . . . . . . . XI

Vorstand 1986-1987 . . . . . . . . . . . . . . . . . . . . . . . . . XI

Vorstand 1985-1986 . . . . . . . . . . . . . . . . . . . . . . . . . XI

Ehrenmitglieder 1986 . . . . . . . . . . . . . . . . . . . . . XI

Ständige Schriftführer . . . . . . . . . . . . . . . . . . . . . XI

Kassenführer . . . . . . . . . . . . . . . . . . . . . . . . . . . XI

Mitglieder des Ausschusses 1986-1987 . . . . . . . . . . . . . . . . . XI

Begrüßungsworte des Vorsitzenden. Zöllner, N. (München) . . . . . . . . . XIII

Theodor-Frerichs-Preis 1986 . . . . . . . . . . . . . . . . . . . . . XVII

Die Erziehung zum Arzt. Zöllner, N. (München) . . . . . . . . . . . . . XIX

\section{Hauptthema:}

\section{Der leise Beginn chronischer Krankheiten - Beiträge zu ihrer Frühsymptomatologie und zur Nosologie der Frühstadien}

Vorsitz: Zöllner N., München; Krück F., Bonn

Einführung. Zöllner, N. (München)

Chronische Glomerulonephritis und chronische interstitielle Nephritis.

Lison, A.-E. (Münster) .

Arterielle Hypertension - Schleichender Risikofaktor für Herz und Gefäße.

Stumpe, K. O. (Bonn) . . . . . . . . . . . . . . . . . . . . . . . . . 6

Herzkrankheiten. Blömer, H. (München) . . . . . . . . . . . . . . . . . 11

Psychoorganische Veränderungen. Stähelin, H. B. (Basel) . . . . . . . . . . 23

Frühsymptomatik und Präventionsmöglichkeiten chronischer Lungenkrankheiten.

l'mer, W. T. (Bochum)

Frühstadien und Verlauf der Wegener'schen Granulomatose.

Goebel, F. D. (München) . . . . . . . . . . . . . . .

Früherkennung und Frühdifferenzierung der Kollagenosen -

Ein Auftrag an den Kliniker. Herzer, $P$. (München) .

Früherkennung schleichender Formen der Hyperthyreose.

Usadel, K. H. (Mannheim)

Paraneoplastische Syndrome - Eine Hilfe bei der Früherkennung

maligner Tumoren? Wilmanns, $W$., Possinger, $K$. (München) .

Störungen des Wasser- und Elektrolythaushaltes - Indikatoren für

neoplastische Prozesse. Kramer, H.J. (Bonn)

Myelodysplasie - Ein neu definiertes Vorstadium akuter Leukämien.

Hunstein, W., Ho, A. D. (Heidelberg) . . . . . . . . . . . . . . . . . . . 85

Vor- und Frühstadien maligner Lymphome. Wilms, K. (Würzburg) . . . . . . . 89

\section{Hauptthema:}

Grundlagenforschung als Voraussetzung medizinischer Fortschritte

\section{Vorsitz: Zöllner, N. (München)}

Einführung. Zöllner, N. (München)

Genetik - Von der Analyse des Vererbungsvorgangs zur synthetischen Biologie.

Hobom, G. (Gießen) 
Gentechnologie - Synthetische Biologie. Winnacker, E. L. (München) . . . . . 105

Immungenetik des Menschen. Riethmüller, G. (München) . . . . . . . . . . 106

Monoklonale Antikörper in der Tumordiagnostik und Tumortherapie. Dörken, B.,

Moldenhauer, G., Momburg, F., Möller, P., Hämmerling, G. J. (Heidelberg) . . . 109

Krebsvirusforschung. zur Hausen, H. (Heidelberg) . . . . . . . . . . . . . 114

Die Wertigkeit einer erhöhten Urinausscheidung modifizierter Nukleoside als

Tumormarker beim Kolonkarzinom. Holstege, A., Pauw, M., Häring, R.,

Kirchner, R., Pausch, J., Gerok, W. (Freiburg)

114

Aktivierung von Protein $\mathrm{C}$ an der Oberfläche kultivierter Endothelzellen -

Die Rolle des Antithrombin III.

Meusel, P., Preissner, K. T., Delvos, U., Müller-Berghaus, G. (Gießen) . . . . . 120

Konservierung von Geweben - Kryopräservation und Kultur.

Federlin, K. (Gießen)

Die Bedeutung zellbiologischer Grundlagenforschung für die Klinik, dargestellt am Beispiel von Biosynthese und Transport des Thyreoglobulins.

Herzog, V. (München) . . . . . . . . . . . . . . . . . . . . . . . . . 134

Möglichkeiten und Grenzen des Lasers. Unsöld, E. (Neuherberg) . . . . . . . 153

Neue bildgebende Systeme. Gudden, F. (Erlangen) . . . . . . . . . . . . . 163

Stoßwellen-Lithotripsie in Niere und Gallenblase. Brendel, W. (München) . . . 168

\section{Hauptthema:}

\section{Gemeinsame Gebiete von Innerer Medizin und Chirurgie}

Vorsitz: Losse, H. (Münster); Bünte, H. (Münster)

Einleitung. Bünte, H. (Münster) . . . . . . . . . . . . . . . . . . . . . 175

Endokrine Hypertonie. Hauss, J., Vetter, H. (Münster) ． . . . . . . . . . . 175

Angiologie aus der Sicht des Chirurgen. Vollmar, J. F. (Ulm) . . . . . . . . . 180

Angiologie aus der Sicht des Internisten. Spengel, F. A. (München) . . . . . . 185

Onkologie. Schwemmle, K. (Gießen) . . . . . . . . . . . . . . . . . . . 192

Zur kombinierten internistisch-chirurgischen Behandlung maligner Tumoren -

aus der Sicht des internistischen Onkologen. Seeber, S. (Leverkusen) . . . . . 202

Organtransplantation. Pichlmayr, R. (Hannover) . . . . . . . . . . . . 206

Nierentransplantation - Internistische Gesichtspunkte.

Fassbinder, W. (Frankfurt/Main) . . . . . . . . . . . . . . . . . . . . 214

Parenterale und Sondenernährung. Hartig, W., Schmoz, G., Weiner, R.,

Matkowitz, R., Grube, U. (Leipzig) . . . . . . . . . . . . . . . . . . . . 224

Parenterale und Sondenernährung. Kleinberger, G. (Steyr) . . . . . . . . . . 235

Rheumatologie. Schöllner, D. (Köln) . . . . . . . . . . . . . . . . . . . 235

Rheumatologie. Schattenkirchner, M. (München) . . . . . . . . . . . . . . 240

\section{Hauptthema, gemeinsam mit der \\ Deutschen Gesellschaft für Ernährung: \\ Ernährung und Krankheit - Aktuelle Probleme und ihre Lösung}

Vorsitz: Wolfram, G. (München); Menden, E. (Gießen)

Einführung. Wolfram, G. (München) . . . . . . . . . . . . . . . . . . . 247

Die Sicherung der Ernährung. Gander, K.-F. (Hamburg) . . . . . . . . . . . 248

Gifte in der Nahrung? Forth, W. (München) . . . . . . . . . . . . . . . . 254

Probleme der Jodprophylaxe. Scriba, P. C., Gutekunst, R., Hötzel, D. (Lübeck) . 263 


\section{Rundtischgespräch: \\ Das koronare Risiko}

Leitung: Zöllner, N. (München)

Einführung. Zöllner, N. (München) . . . . . . . . . . . . . . . . . . . 303

Hypertonie. Distler, A. (Berlin) . . . . . . . . . . . . . . . . . . . . . 307

Diabetes mellitus. Sailer, D. (Erlangen) . . . . . . . . . . . . . . . . . 311

Rolle von Fettstoffwechselstörungen. Schlierf, G. (Heidelberg) . . . . . . . . 316

Rauchen als Faktor? Seidel, D. (Göttingen) . . . . . . . . . . . . . . . . 320

\section{Rundtischgespräch:}

\section{AIDS - Entwicklungen seit 1984}

Leitung: Goebel, F.-D. (München); Hehlmann R. (München)

Definitionen, Klinik und Epidemiologie der HTLV-III-assoziierten Erkrankungen.

Lüthy, R. (Zürich) .

Klinik und Behandlung des Kaposi-Sarkoms bei erworbenem Immunmangel-

Syndrom (AIDS). Braun-Falco, O., Brunner, R. (München)

Klinik und Therapie maligner Lymphome bei AIDS-Symptomatologie der

AIDS-Enzephalopathie. Huhn, D. (Berlin) . . . . . . . . . . . . . . . . 330

Diagnostik der HTLV-III-Infektion: Tests, Infektionsschutz.

Hehlmann, R. (München) .

Behandlung opportunistischer Infektionen bei AIDS.

Helm, E. B. (Frankfurt/Main) .

Möglichkeiten der Impfprophylaxe und virustatischen Therapie der

HTLV-III/LAV-Infektion. Goebel, F. -D. (München)

Gesundheitspolitische Maßnahmen und Aspekte zur Eindämmung der

HTLV-III-Infektion. Steinbach, M. (Bonn).

\section{Rundtischgespräch:}

\section{Therapeutische Grenzgebiete von Innerer Medizin und Chirurgie an Beispielen aus der Gastroenterologie}

\section{Leitung: Demling, L. (Erlangen); Bünte, H. (Münster)}

Einführung/Zusammenfassung. Demling, L. (Erlangen) . . . . . . . . . . . 346

Krankheiten der Speiseröhre. Tytgat G. N. (Amsterdam) . . . . . . . . . . . . . . 347

Krankheiten der Speiseröhre. Siewert, J. R. (München) . . . . . . . . . . . . . . . . . 349

Ulkuskrankheit - Standpunkt des Internisten. Domschke, W. (Erlangen) . . . . 352

Ulkuskrankheit - Standpunkt des Chirurgen. Langhans, P. (Münster) . . . . . 356

Entzündliche und neoplastische Krankheiten des Dickdarms.

Frühmorgen, $P$. (Ludwigsburg) 
Entzündliche und neoplastische Krankheiten des Dickdarms.

Arnold, K. (Wiesbaden)

Internistische Aspekte der Krankheiten der Gallenwege und des Pankreas.

Lux, G. (Erlangen) . . . . . . . . . . . . . . . . . . . . . . . . . . 367

Krankheiten der Gallenwege und des Pankreas. Lingemann, B. (Münster) . . . 369

ERCP und endoskopische Sphinkterotomie bei akuter Pankreatitis. Kautz, G.,

Bünte, H. (Münster) . . . . . . . . . . . . . . . . . . . . . . . . . . 37

\section{Symposium A:}

\section{Angeborene Störungen des Lipidstoffwechsels - Pathogenese und Therapie}

Vorsitz: Lewis, B. (London); Keller, Ch. (München)

Introduction. Lewis, B. (London)

Familiäre Hypercholesterinämie

Neuere Erkenntnisse zur Pathogenese der Familiären Hypercholesterinämie.

Beisiegel, U., Weber, W. (Hamburg) . . . . . . . . . . . . . . . . .

Neue experimentelle Aspekte zur Pathogenese der Arteriosklerose bei Familiärer

Hypercholesterinämie. Dresel, H. A. (Heidelberg)

Molekulargenetik

DNA Deletions and DNA Polymorphisms of Low Density Lipoprotein (LDL)

Receptor Gene - Their Use in Diagnosis. Humphries, S. E., Dunning, A.,

Taylor, R., Kessling, A., Horsthemke, B. (London)

Enzymdefekte

Genetisch bedingte Funktionsstörungen lipolytischer Enzyme und ihre Auswirkung

auf die Plasmalipoproteine. Greten, H. (Hamburg) . . . . . . . . . . . . . 402

Störungen des Apolipoprotein-Metabolismus

Mutants of Apolipoproteins A and C. Sirtori, C. R., Louati, M. R. (Milano) . . . 403

Mutanten des Apolipoprotein E und Familiäre Dysilipoproteinämie.

Utermann, G. (Innsbruck) . . . . . . . . . . . . . . . . . . . . . . . . 412

\section{Therapie}

Hyperlipidaemia: Screening and Therapy. Lewis, B. (London) . . . . . . . . 412

Plasmaaustausch und verwandte Therapieformen bei schwerer

Familiärer Hypercholesterinämie. Keller, Ch. (München) ． . . . . . . . . . 419

\section{Symposium B:}

Chance und Risiko - Alternativen zwischen palliativer und kurativer Therapie

Vorsitz: Heimpel, H. (Ulm); Anschütz, F. (Darmstadt)

Einführung. Heimpel, H. (Ulm) 
Konventionelle Behandlung versus Knochenmarktransplantation bei chronisch-myeloischer Leukämie

Prognose und Verlauf bei konventioneller Behandlung. Hehlmann, R. (München) 426 Prognose und Verlauf bei Knochenmarktransplantation. Ostendorf, P. (Hamburg) 431

Wann und bei welchen Patienten sollen Herzklappenfehler chirurgisch korrigiert werden?

Internistische Gesichtspunkte. Lichtlen, P. (Hannover) . . . . . . . . . . . 435

Chirurgische Gesichtspunkte. Bircks, W. (Düsseldorf) . . . . . . . . . . . . 435

Therapeutische Strategien bei kleinzelligem Bronchialcarcinom

Chance der Lebensverlängerung und Minderung der Lebensqualität durch

intensive Chemotherapie. Schreml, W. (Günzburg) . . . . . . . . . . . . .

Rundtischgespräch zum Thema des Symposiums

Leitung: Heimpel, H. (Ulm); Anschütz, F. (Darmstadt)

Darstellung der Prognose aus der Sicht des Biostatistikers. Neiss, A. (Innsbruck) . 446

Die Information des Patienten - ein Sprachproblem. Raspe, H. H. (Hannover) . 448

Die emotionale Situation im Entscheidungsprozeß. Köhle, K. (Köln) . . . . . 452

\section{Symposium C: \\ Gicht und andere Störungen des Purin- und Pyrimidinstoffwechsels}

Vorsitz: Kelley, W. N. (Ann Arbor); Gröbner, W. (Balingen)

Einführung. Gröbner, W. (Balingen)

455

Metabolische Aspekte zur Pathogenese der Hyperurikämie.

Gutensohn, W. (München) . . . . . . . . . . . . . . . . . . . . . . . 456

Renale Aspekte zur Pathogenese der Hyperurikämie. Deetjen, P. (Innsbruck) . . 461

HGPRT Deficiency - The Molecular Basis of the Clinical Syndromes.

Kelley, W. N., Searle, J. G., Wilson, J. W. (Ann Arbor) . . . . . . . . . . . 465

Klinisches Bild und Diagnose der Gicht. Schattenkirchner, M. (München) . . . 469

Ernährungstherapie der Hyperurikämie - Wissenschaftliche Grundlagen und praktische Konsequenzen. Löffler, $W$. (München) . . . . . . . . . . . . . 484

Arzneimitteltherapie der Hyperurikämie. Gröbner, W. (Balingen) . . . . . . . 492

When to Treat Hyperuricaemia.

Woods, H. F., Bax, N. D. S., Jackson, P. R. (Sheffield) . . . . . . . . . . . . . 497

2,8-Dihydroxyadenine Lithiasis - Epidemiology, Pathogenesis and Therapy.

Simmonds, H. A. (London) . . . . . . . . . . . . . . . . . . . . . . . 503

Myopathie bei AMP-Deaminase-Mangel. Pongratz, D. E. (München) . . . . . 508

Störungen des Pyrimidinstoffwechsels. Reiter, S. (München) . . . . . . . . . 512

\section{Symposium D: \\ Der Typ II Diabetes}

\section{Vorsitz: Schöffling, K. (Frankfurt/Main)}

Einführung und Begründung des Themas. Schöffling, K. (Frankfurt/Main) . . . 521

Klassifikation und Vererblichkeit der Zuckerkrankheit.

Köbberling, J. (Wuppertal) 
Epidemiology of Diabetes. Skrabalo, Z., Aganović, I. (Zagreb) . . . . . . . . 532

New Aspects of Pathogenesis of Type II Diabetes.

Vranic, M., Efendic, S. (Toronto, Stockholm) ～. . . . . . . . . . . . . . 546

Die Differentialtherapie des Typ-II-Diabetes.

Althoff, P.-H., Rosak, C. (Frankfurt/Main) . . . . . . . . . . . . . . . . 559

Fettstoffwechsel bei Typ II Diabetes mellitus.

Gries, F. A., Koschinsky, T. (Düsseldorf) . . . . . . . . . . . . . . . . . 571

Die Angiopathien des Typ II Diabetes. Standl, E. (München) . . . . . . . . . 579

\section{Symposium E: \\ Polyenfettsäuren und Eikosanoide - Ihre klinische Bedeutung heute}

Vorsitz: Wolfram, G. (München)

Einführung. Wolfram, G. (München) . . . . . . . . . . . . . . . . . . . 587

Struktur und Zufuhr von Polyenfettsäuren. Billek, G. (Hamburg) . . . . . . $\quad 588$

Nahrungsfett und Serumlipoproteine. Schwandt, P. (München) . . . . . . . . 595

Polyenfettsäuren und Prostaglandinbiosynthese. Adam, $O$. (München) . . . . . 600

Eicosanoide und essentielle Fettsäuren im kardiovaskulären System.

Schrör, K. (Köln) . . . . . . . . . . . . . . . . . . . . . . . . . . . 610

Eicosanoide und periphere Verschlußkrankheit. Sinzinger, H. (Wien) . . . . . 619

Eicosanoide und Magen-Darm-Trakt. Peskar, B. M. (Bochum) . . . . . . . .623

Eicosanoide und Lungenfunktion. Peskar, B. A., Simmet, Th. (Bochum) . . . . 631

\section{Symposium F: \\ Wahl der Nahrungsproteine - Grundlagen und Diätetik}

Vorsitz: Barth, C. A. (Kiel); Fürst, P. (Stuttgart)

Einführung. Barth, C. A. (Kiel) _ . . . . . . . . . . . . . . . . . . . . 639

Some Aspects of In Vivo Human Protein and Amino Acid Metabolism, with Particular Reference to Nutritional Modulation. Young, V. R.,

Fukagawa, N. (Cambridge)

Are There Atherogenic Dietary Proteins?

West, C. E., Beynen, A. C. (Wageningen, Utrecht) . . . . . . . . . . . . . 666

The Optimum Form of Dietary Nitrogen in Gastrointestinal Disease: Proteins,

Peptides or Amino Acids? Grimble, G. K., Silk, D. B. A. (London) . . . . . . .674

Protein- und Aminosäurenstoffwechsel bei Leberinsuffizienz - Infusions-

therapeutische und diätetische Folgerungen. Holm, E., Leweling, H., Staedt, U.,

Striebel, J.-P., Tschepe, A., Uhl, W. (Mannheim) . . . . . . . . . . . . . . 685

Nahrungsprotein und Nierenkrankheiten. Fürst, P. (Stuttgart) . . . . . . . . 737

Nahrungsprotein und die Resorption von Mineralien und Spurenelementen,

insbesondere Eisen. Hallberg, L. (Göteborg) . . . . . . . . . . . . . . . . 743

Namenverzeichnis . . . . . . . . . . . . . . . . . . . . . . 750

Sachverzeichnis . . . . . . . . . . . . . . . 752 


\section{Vorsitzender}

1986-1987 Prof. Dr. med. Dr. h.c. mult. E. F. Pfeiffer - Ulm

1986-1987 Prof. Dr. med. Dr. h.c. mult. E. F. Pfeiffer - Ulm

Prof. Dr. med. N. Zöllner - München

Prof. Dr. med. L. Demling - Erlangen

Prof. Dr. med. E. Wetzels - Rosenheim

Prof. Dr. med. K. Miehlke - Wiesbaden

Prof. Dr. med. H. Schmidt - Wiesbaden

\section{Vorstand}

1985-1986 Prof. Dr. med. N. Zöllner-München

Prof. Dr. med. F. Anschütz - Darmstadt

Prof. Dr. med. Dr. h.c. mult. E. F. Pfeiffer-Ulm

Prof. Dr. med. L. Demling - Erlangen

Prof. Dr. med. K. Miehlke - Wiesbaden

Prof. Dr. med. H. Schmidt - Wiesbaden

1986 Ehrenmitglieder

Prof. Dr. med. H. Gillmann - Limburgerhof

Prof. Dr. med. F. Krück-Bonn

Prof. Dr. med. G. A. Neuhaus - Berlin

\section{Ständige Schriftführer}

1961-1984 Prof. Dr. med. B. Schlegel-Wiesbaden

ab 1984 Prof. Dr. med. K. Miehlke - Wiesbaden

\section{Kassenführer}

1967-1984 Prof. Dr. med. K. Miehlke-Wiesbaden

ab 1984 Prof. Dr. med. H. Schmidt - Wiesbaden

\section{Mitglieder des Ausschusses}

1986-1987 Prof. Dr. med. G. Geyer - Wien

Prof. Dr. med. P. C. Scriba - Lübeck

Prof. Dr. med. K. Schöffling - Frankfurt

Prof. Dr. med. P. G. Scheurlen - Homburg

Prof. Dr. med. E. Wetzels - Rosenheim

Prof. Dr. med. U. C. Dubach - Basel

Prof. Dr. med. W. Wilmanns - München

Prof. Dr. med. M. Classen - München

Prof. Dr. med. G. Paumgartner - München

Prof. Dr. med. H. Löffler - Kiel

Prof. Dr. med. H. Goebell - Essen

Prof. Dr. med. K. Federlin - Gießen

Prof. Dr. med. W. T. Ulmer - Bochum

Prof. Dr. med. H. Greten - Hamburg

Prof. Dr. med. H. Schliack - Hannover

Prof. Dr. med. H. -P. Schuster - Hildesheim

Prof. Dr. med. W. Waldhäusl-Wien

Prof. Dr. med. $H$. Kaess - München

Prof. Dr. med. K. Wilms - Würzburg

Prof. Dr. med. A. Distler - Berlin

Prof. Dr. Dr. med. K. -H. Meyer zum Büschenfelde - Mainz

Prof. Dr. med. G. Riecker - München

Prof. Dr. med. D. L. Heene - Mannheim

Prof. Dr. med. D. Reinwein - Essen

Prof. Dr. med. H. Losse-Münster 


\section{Probleme der Jodprophylaxe}

Scriba, P. C., Gutekunst, R., Hötzel, D. (Klinik für Innere Medizin der Medizinischen Hochschule Lübeck)

Voraussetzung für eine umfassende Prophylaxe ist, daß die zu vermeidende Gesundheitsstörung in einer ernstzunehmenden Häufigkeit vorliegt. Es ist ferner zu fordern, daß sie klinisch und damit auch volkswirtschaftlich bedeutsam ist und daß die vorgesehenen Maßnahmen der Krankheit zuverlässig vorbeugen und bezahlbar sind. Schließlich darf die Prophylaxe selbst kein oder kein unvertretbares Risiko mit sich bringen. Nach Besprechung dieser Voraussetzungen werden die lebensmittelrechtliche Situation in der Bundesrepublik dargestellt und anschließend Folgerungen und Forderungen formuliert.

\section{Epidemiologie}

Die „European Thyroid Association“ hat 1985 eine jahrelange epidemiologische Datensammlung publiziert [26, 27]. Bei aller möglichen Kritik lassen sich drei Aussagen festhalten (Abb. 1):

1. In Zentraleuropa gibt es, insbesondere mit der Bundesrepublik und der DDR, ein endemisches Strumagebiet, in dem definitionsgemäß mehr als 10\% der gesamten Bevölkerung von einer Schilddrüsenvergrößerung betroffen sind.

2. In Südeuropa findet man zum Teil schwere Strumaendemien vorwiegend regional, wie bezeichnet.

3. Einige Länder haben eine sehr effektive Jodprophylaxe, so z. B. Schweden, Finnland, die CSSR, die Schweiz und Bulgarien.

In der Bundesrepublik stützte sich die epidemiologische Argumentation auf die 5,4 Millionen Rekruten, deren Musterungsunterlagen von Horster et al. 1975 ausgewertet wurden [19] und bei denen im Mittel für 15\% das Vorliegen einer Struma anzunehmen 


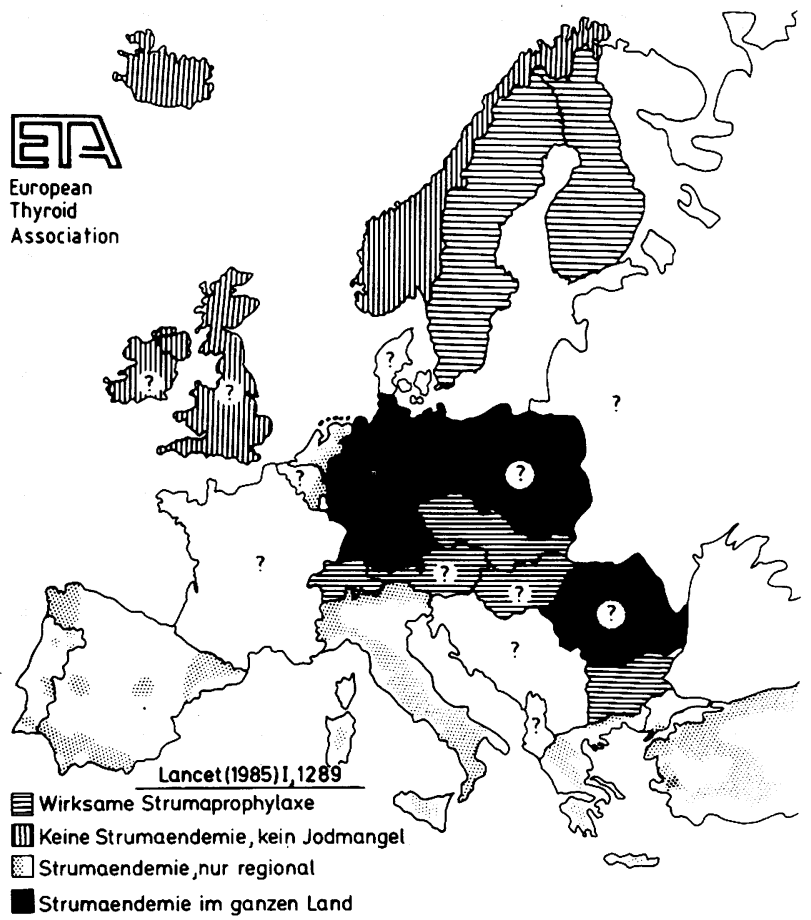

Abb. 1. Übersicht über Strumaprävalenz, Jodmangel und Strumaprophylaxe der European Thyroid Association, nach Scriba et al. [26]

war. Nach den epidemiologischen Empfehlungen der WHO hat man in solchen Gebieten Schulkinder zu untersuchen [28]. Bei knapp 2000 Schulkindern aus der ganzen Bundesrepublik ermittelte die Sektion Schilddrüse schon 1975 palpatorisch eine Schilddrüsenvergrößerung bei 32\% der Knaben und 42\% der Mädchen [12].

Seit 1981 steht nun die Methode der sonographischen Volumenbestimmung [4] auch für epidemiologische Untersuchungen zur Verfügung. Breite, Tiefe und Länge des Schilddrüsenlappens miteinander und mit dem Korrekturfaktor von knapp 0,5 multipliziert, ergeben das Volumen in ml. Auf der Suche nach einem für die Ermittlung des normalen Schilddrüsenvolumens geeigneten Kollektiv, mußten wir die Bundesrepublik verlassen. Schweden hat im ganzen eine ausreichende Jodversorgung [26, 27], vorwiegend aufgrund einer generellen Kochsalzjodierung, so daß die WHO-Empfehlung einer täglichen Aufnahme von 150 bis $300 \mu \mathrm{g}$ Jod erfüllt wird [23, 28]. Und hier in Schweden findet man normale mittlere Schilddrüsenvolumina, nicht aber in der Bundesrepublik. So haben 13jährige Schulkinder in Stockholm eine Schilddrüsengröße von 4,2 $\mathrm{ml}$ und eine Jodausscheidung von $130 \mu \mathrm{g} / \mathrm{g}$ Kreatinin (Medianwerte). In der Bundesrepublik ist die Schilddrüse gut doppelt so groß bei einer Jodausscheidung von nur $39 \mu \mathrm{g} / \mathrm{g}$ Kreatinin [8-11].

Auch bei Erwachsenen ist die Schilddrüse in Schweden mit $9 \mathrm{ml}$ nur halb so groß wie in der Bundesrepublik, während umgekehrt die Jodausscheidung in Stockholm gut doppelt so hoch wie in der Bundesrepublik ist $[10,11]$.

Wenn man nun den schwedischen Mittelwert des Schilddrüsenvolumens plus zwei Standardabweichungen als obere Grenze des normalen Schilddrüsenvolumens nimmt, so gibt Abbildung 2 wieder, daß im Norden der Bundesrepublik etwa 30 bis $40 \%$ der 13jährigen Kinder eine vergrößerte Schilddrüse zeigen. Südbaden und das Bodenseegebiet haben relativ günstige Werte, was wohl weniger an der Ausstrahlung der Schweiz als mehr daran liegt, daß hier in einigen Bereichen bis heute noch in den Schulen Jodtabletten ausgeteilt werden. In Mitteldeutschland und vor allem in Bayern 


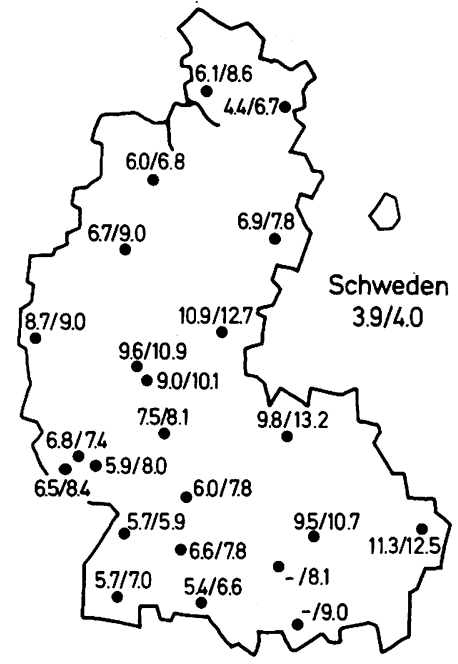

Schilddrüsenvolumen (Median, $\mathrm{ml}$ ) bei 2224 13-jährigen $\sigma^{2} / q$
Abb. 2. Sonographisch ermittelte Schilddrüsenvolumina bei 13jährigen Schulkindern; Vergleich der Bundesrepublik mit Stockholm, nach Gutekunst et al. [8]

ist das Problem rund doppelt so schlimm mit 50, 60 oder $70 \%$ der Kinder, die eine vergrößerte Schilddrüse zeigen [10,11]. - Zugleich müssen wir uns aber fragen, welchen Krankheitswert diese erschreckende Prävalenz der vergrößerten Schilddrüse aufweist.

\section{Gesundheitliche Relevanz}

Ich möchte die medizinische Bedeutung des epidemiologischen Problems anhand von fünf verschiedenartigen Befunden aufzeigen. Zum ersten erlaubte die sonographische epidemiologische Untersuchung $[10,11]$ zu zeigen, daß in Schweden nur $5,6 \%$ der Schilddrüsen abnormale Echomuster aufweisen, während dies in der Bundesrepublik bei 16\% der Probanden der Fall war (Tabelle 1). Diese Schilddrüsen sind also nicht nur vergrößert, sie sind auch strukturell verändert [10,11].

Zweitens, die mechanischen Auswirkungen der Struma sind jedem Mediziner bekannt [23]. Frey, K. W. et al. [6] zeigten, daß Strumen der WHO-Grade Ib und II in 9 bzw. 25\% röntgenologisch eine Einengung der Trachea um die Hälfte aufweisen. Diese

Tabelle 1. Echostrukturveränderungen bei Erwachsenen, aus Gutekunst (11)

\begin{tabular}{lcc}
\hline Echostruktur & $\begin{array}{l}\text { Schweden } \\
(\mathrm{n}=303)\end{array}$ & $\begin{array}{c}\text { Deutschland } \\
(\mathrm{n}=1397)\end{array}$ \\
\hline Normal & $94,4 \%$ & $84,0 \%$ \\
Diffus echoverarmt & $1,0 \%$ & $2,5 \%$ \\
Diffus echokomplex & - & $0,1 \%$ \\
Uni- und/oder multifokale Veränderungen & $2,6 \%$ & $13,4 \%$ \\
Zysten & $1,3 \%$ & $5,9 \%$ \\
Verkalkungen & $0,3 \%$ & $2,1 \%$ \\
Echoarme Knoten & $0,3 \%$ & $3,2 \%$ \\
Echoreiche Knoten & $0,7 \%$ & $2,1 \%$ \\
Echokomplexe Knoten & - & $0,1 \%$ \\
\hline
\end{tabular}

Gutekunst et al.: Acta Endocr (Kbh) 1986. 
auch bei verhältnismäßig kleinen Strumen bereits nachweisbaren Trachealstenosen bedeuten auf dem Umweg über die vermehrte Atemarbeit eine Belastung des kleinen Kreislaufs, an die häufig nicht gedacht wird.

Zum dritten sind die funktionellen Auswirkungen der Jodmangelstruma zu bedenken. Niemand behauptet, daß es in der Bundesrepublik eine endemische Form von Kretinismus gäbe. Aber es liegen Untersuchungen über die gefährlich niedrige Jodausscheidung bei Neugeborenen vor [15, 32]. Die Göttinger Gruppe um Stubbe und Heidemann [16] fand bei rund einem Prozent der Neugeborenen das Vorliegen einer Struma neonatorum [31], zum Teil mit Hypothyreose. Diese Tatsache ist an der hohen Rate der falsch positiven Befunde des TSH-Screenings auf Neugeborenenhypothyreose in der Bundesrepublik schuld [32]. Die Autoren haben ferner die von Teller in Ulm [18] gezeigte Verzögerung der Knochenkernreifung bei Struma neonatorum bestätigt und überdies auf die Möglichkeit der verlangsamten EEG-Reifung [16] hingewiesen. Ich möchte diese Befunde nicht dramatisieren, muß aber darauf hinweisen, daß der Jodmangel an unseren Neugeborenen keineswegs spurlos vorübergeht.

Die vierte medizinisch bedeutsame Folge der Jodmangelstruma ist in dem Gebiet der Schilddrüsenautonomie aufzufinden. Während in den Vereinigten Staaten nur wenige Prozent der Patienten mit Hyperthyreose diese Hyperthyreose auf dem Boden einer Schilddrüsenautonomie haben [34], sind dies in der Bundesrepublik mehr als die Hälfte aller hyperthyreoten Patienten [14, 27]. Es sind sowohl das solitäre autonome Adenom als auch die multifokale oder disseminierte Autonomie in der Bundesrepublik viel häufiger, und dies ist, wie aus den Studien der Gruppe um Studer in Bern [7] und Emrich in Göttingen [5] hervorgeht, eine indirekte Folge des Jodmangels im Sinne der sogenannten fehlerhaften Anpassungshyperplasie. Die Schilddrüsenautonomie ist eine Krankheit der alten Menschen, und diese sind wiederum besonders gefährdet durch thyreotoxische Exazerbationen $[3,13,17]$ bei exzessiver, aber nur bei exzessiver Jodbelastung.

Der fünfte und letzte Befund betrifft die Struma maligna. Diese ist, wie vor allem von Hedinger und Mitarbeitern [2, 24] wiederholt ausgeführt, im Jodmangelgebiet zwar nicht unbedingt häufiger, die Struma maligna tritt aber vermehrt in ihrer bösartigen Form als anaplastisches oder auch follikuläres Karzinom auf. Die histologische Nachuntersuchung zeigte, daß in den Jahren des Jodmangels das gutartigere papilläre Schilddrüsenkarzinom mit $7,8 \%$ in der Schweiz seltener war und sein Anteil nach Jodprophylaxe auf 33,4\% zu Lasten der bösartigen Tumoren anstieg [2].

\section{Kostenrelevanz}

Horster et al. haben berichtet [20], daß allein die gesetzlichen Krankenkassen im Jahre 1981750 Mill. DM an Gesamtkosten einschließlich Diagnostik und Therapie für die Jodmangelstruma ausgegeben haben. Dieser Betrag läßt sich nahezu beliebig steigern, wenn die nicht mehr übersehbare Ärzteschwemme sich vermehrt mit diesem epidemiologischen Problem beschäftigt. Umgekehrt würde die Jodprophylaxe praktisch nichts kosten.

\section{Wirksamkeit der Jodprophylaxe}

Die Wirksamkeit der Jodprophylaxe ist in der ganzen Welt und vor allem auch in unseren unmittelbaren Nachbarländern gut dokumentiert. Ich nenne noch einmal die Länder Finnland, Schweden, CSSR, Schweiz und verzichte darauf, die in der Literatur zugängigen Einzelbeweise für die Wirksamkeit der Jodprophylaxe hier darzustellen [23, 26-28]. Ich weise nur darauf hin, daß die in den meisten Ländern schrittweise eingeführte Jodprophylaxe, so z. B. in der Schweiz und in der CSSR, zum Teil erst in jüngster 
Zeit optimale Wirksamkeit erreicht hat. Dies hat zur Folge, daß man in der älteren Bevölkerung dieser Länder auch heute noch die Reste der ehemaligen Jodmangelstrumaendemie findet.

\section{Risiko?}

Die Frage des sogenannten Jod-Basedow spielte im Gegensatz zu heute vor allem früher bei der Diskussion über die Einführung einer Strumaprophylaxe mit jodiertem Speisesalz eine große Rolle [23]. Die Beobachtungen in Tasmanien zeigen, worum es immer wieder geht [29]. Hier wurde durch verschiedene Maßnahmen die alimentäre Jodzufuhr um etwa $300 \mu \mathrm{g}$ pro Tag erhöht. In der Folge kam es zu einer Zunahme der Hyperthyreoserate von 0,3 auf $1,2 \%$. Diese Zunahme war auf wenige Jahre beschränkt, d.h. also passager. Noch wichtiger ist, daß sie praktisch ausschließlich Patienten jenseits des 40 . Lebensjahres betraf und, wie in weiteren Studien gezeigt wurde, Kranke mit immunogener Hyperthyrose vom Typ des Morbus Basedow nicht häufiger wurden [1]. Daraus kann man schließen, daß es sich also um Patienten mit Schilddrüsenautonomie gehandelt hat, bei denen die genannte Joddosis eine Vorverlegung der Manifestation der Hyperthyreose zur Folge hatte. Zusammengefaßt kann man dieses Problem folgendermaßen darstellen [23]: Man kann einen Morbus Basedow durch Jodmangel nicht verhindern, der Morbus Basedow muß vielmehr in jedem Falle behandelt werden. Desgleichen muß ein bekanntes autonomes Adenom behandelt werden, weil es sonst im Alter mit hoher Wahrscheinlichkeit zu thyreotoxischen Entgleisungen führt. Damit verbleibt die Frage, ob bei multifokaler oder disseminierter Schilddrüsenautonomie mit Euthyreose durch Jodsalzprophylaxe eine Hyperthyreose hervorgerufen werden kann.

Genau diese Frage hat Joseph untersucht [21]. Er hat die gewünschten $100 \mu \mathrm{g}$ Jod in Form von Tabletten Patienten gegeben, die nachweislich eine genügende Menge autonomen Schilddrüsengewebes hatten. Dabei blieben die FTE- und $T_{3}$-Werte im Normbereich, der TSH-Anstieg wurde supprimiert und klinisch blieben diese Patienten euthyreot. Man kann also folgern, daß es bei der niedrig dosierten Prophylaxe mit jodiertem Speisesalz kein Hyperthyreoserisiko gibt. Man sollte auch nicht vergessen, daß die Inzidenz der Formen von Schilddrüsenautonomie, welche die Patienten gegen höhere Joddosen empfindlich machen, gerade durch die Prophylaxe mit jodiertem Speisesalz vermindert wird, so daß diese schließlich praktisch verschwinden [1,23].

\section{Lebensmittelrechtliche Situation}

Im Jahr 1981 hat der Gesetzgeber nach langem Zögern eine Verbesserung der sogenannten „Diätverordnung“ verkündet [25]. Für die Strumaprophylaxe mit jodiertem Speisesalz brachte diese Veränderungen

- den Wegfall des Warnhinweises ,nur bei ärztlich festgestelltem Jodmangel“ zugunsten der erlaubten Aussage ,,geeignet zur Verhütung und Behandlung von Jodmangel",

- die Verbesserung der Stabilität durch die Zulassung von Natrium- oder Kaliumjodat anstelle von Jodid und

- die Anhebung des Jodgehaltes der jodierten Speisesalze auf 15 bis $25 \mathrm{mg} / \mathrm{kg}$, das sind im Mittel $20 \mathrm{mg} / \mathrm{kg}$ Kochsalz.

Wie wichtig die Stabilitätsfrage ist, konnte $\mathrm{H}$. Bürgi wiederum im Rahmen der ETA belegen [26], als er in 104 Salzproben aus 19 Ländern fand, daß der Jodgehalt in 30\% der Fälle um mehr als 30\% den Sollwert unterschritt. In der Bundesrepublik besteht dieses Problem zum Glück nicht. 
Trotz dieser seinerzeitigen Verbesserungen haben wir mit dem jodierten Speisesalz ein dreifaches Dilemma. Zum ersten blieb es bei dem übertriebenen Freiwilligkeitsprinzip, welches voraussetzt, daß jeder vom Jodmangel Gefährdete über diese Zusammenhänge aufgeklärt werden kann. Eine wirksame Aufklärung der Bevölkerung ist bisher nicht erfolgt. Hier ist das BMJFG in einer Bringschuld, da bei den seinerzeitigen Beratungen im Bundesgesundheitsrat, als man sich für die Freiwilligkeit entschied, versprochen wurde, sich für die Aufklärung der Bevölkerung zu engagieren. Leider ist es im wesentlichen bei diesem Versprechen geblieben.

Den Bemühungen des „Arbeitskreises Jodmangel“, der von Mitgliedern der beiden DGEs, den „Deutschen Gesellschaften für Ernährung und Endokrinologie“, gegründet wurde, ist zu verdanken, was bisher erreicht wurde. Die jodierten Speisesalze sollen im besser aufgeklärten Süden der Bundesrepublik inzwischen 30 bis 40\%, im Norden dagegen nur 10 bis 20\% Marktanteile erreicht haben. Wir sind also noch weit von einer möglichst vollständigen Erfassung der Bevölkerung entfernt.

Mehr als fünf Hersteller produzieren heute in der Bundesrepublik jodiertes Speisesalz. Sie müssen auf den Hinweis, ,jodiertes Salz" achten und wissen, daß einfache Meersalze ungeeignet sind, weil sie solange gereinigt werden, bis das ursprünglich vorhandene Jod entfernt ist. Das wichtigste natürliche Nahrungsmittel mit ausreichendem Jodgehalt ist der Seefisch, den man aber nahezu täglich essen müßte, um den alimentären Jodmangel auszugleichen. Jodidtabletten a $100 \mu \mathrm{g}$ sind eine Alternative. Diese Jodidtabletten kommen in Betracht, wenn jodiertes Salz nicht ausreichend benutzt werden kann. Herr Teller in Ulm [30] hat erreicht, daß in seinem Einzugsbereich alle Schwangeren während der Schwangerschaft täglich 100 bis $200 \mu$ g Jodid in Tablettenform einnehmen, und damit die früher recht häufige Struma neonatorum im Umkreis der Universität Ulm praktisch ausgerottet. Es wäre echter Fortschritt, wenn alle Schwangeren in der Bundesrepublik diese Jodidtabletten erhielten.

Aber nicht nur hinsichtlich der Freiwilligkeit, sondern auch hinsichtlich der Menge besteht ein Dilemma mit dem jodierten Speisesalz. Bei dem Jodgehalt von $20 \mathrm{mg} / \mathrm{kg}$ muß man täglich $5 \mathrm{~g}$ zusalzen, wenn man die gewünschten zusätzlichen $100 \mu \mathrm{g}$ Jod pro Tag zuführen will. Diese Zusalzmenge von $5 \mathrm{~g}$ pro Tag ist heute wohl nicht mehr realistisch. Dies ist wahrscheinlich ein Erfolg der Bemühungen der Hochdruckliga um Senkung des Kochsalzkonsums, den wir begrüßen, der uns aber zu Konsequenzen zwingt. Das Dortmunder Forschungsinstitut für Kinderernährung hat Familienuntersuchungen angestellt $[22,33]$. Zusammengefaßt zeigen sie, daß die Zusatzmenge im Haushalt in mehr als der Hälfte der Fälle unter $1,7 \mathrm{~g}$ pro Kopf und Tag liegt, wobei ein Teil dieses Salzes auch noch mit dem Kochwasser verloren geht. Auch aus diesem Grunde wird es unwahrscheinlicher, daß man die Ziele der Jodprophylaxe mit dem Zusalzen alleine erreichen kann.

Und das dritte Problem der Diätverordnung stellen alle die Menschen dar, die weitgehend von Gemeinschaftsverpflegung oder Fertignahrungsmittel zu leben haben. Die Benutzung von jodiertem Speisesalz in der Gemeinschaftsverpflegung ist nicht praktikabel, da nach geltendem Recht, diese dann als diätetisches Lebensmittel angeboten werden müßte. Ich wiederhole auch hier meinen früheren Hinweis, daß ich geradezu darauf warte, daß ein Rekrut wegen Wehrdienstbeschädigung klagt, weil seine Struma an Größe zunahm, während er in der Bundeswehr gezwungen war, ohne jodiertes Speisesalz zu essen. Ich vermute, daß dieser Rekrut gewinnen würde.

\section{Folgerungen und Forderungen}

Zum ersten sollte unser Regelsalz jodiert sein, derart, daß man, wenn man Salz im Laden verlangt, automatisch jodiertes Salz erhält. Nichtjodiertes Kochsalz soll vorrätig, aber nur auf ausdrücklichen Wunsch erhältlich sein. Dies wäre eine Umkehrung der gegenwärtigen Situation, welche z. B. der Regelung in der Schweiz entspräche. 
Zum zweiten müssen für Gemeinschaftsverpflegung und Fertignahrungsmittel juristische Wege gefunden werden, die eine Herstellung mit jodiertem Speisesalz zulassen. Dabei sollte eine Deklarationspflicht als diätetisches Lebensmittel ausgeschlossen sein.

Zum dritten bitte ich alle anwesenden Kolleginnen und Kollegen, die Vertreter der Medien und die Verantwortlichen in Politik und Verwaltung, das Ihre beizutragen, damit unsere Bevölkerung darüber aufgeklärt wird, wie überflüssig die Jodmangelkrankheiten einschließlich der Struma sind und wie leicht und billig der Jodmangel in der Nahrung zu beheben wäre.

Zum Schluß mache ich auf die Notwendigkeit aufmerksam, daß die Jodprophylaxe langfristig epidemiologisch überwacht werden muß.

\section{Literatur}

1. Adams DD, Kennedy TH, Stewart JC, Utiger RE, Vidor GI (1975) Hyperthyreoidism in Tasmania following iodine supplementation: Measurements of thyroid-stimulating autoantibodies and thyrotropin. J Clin Endocrinol Metab 41:221-228 - 2.Bubenhofer R, Hedinger Chr (1977) Schilddrüsenmalignome vor und nach Einführung der Jodsalzprophylaxe. Schweiz med Wochenschr 107:733-741 - 3. Belfiore A, Sava L, Runello F, Tomaselli L, Vigneri R (1983) Solitary autonomously functioning thyroid nodules iodine deficiency. J Clin Endocrinol Metab 56:283-287 - 4 . Brunn J, Block U, Ruf G, Bos I, Kunze WP, Scriba PC (1981) Volumetrie der Schilddrüsenlappen mittels Real-time-Sonographie. Dtsch med Wochenschr. 106:1338-1340 - 5. Emrich D, Bähre M (1978) Autonomy in euthyroid goitre: Maladaption to iodine deficiency. Clin Endocr 8:257-2656. Frey KW, Engelstädter M (1976) Kropfhäufigkeit und Tracheal-Einengung im poliklinischen Krankengut Münchens. Münch med Wochenschr 118:1555-1559 - 7. Gerber H, Peter HJ, Ramelli F, Miloni E, König MP, Studer H, Berchtold R, Gemsenjäger E (1983) Autonomie und Heterogenität der Follikel in der euthryreoten und hyperthyreoten menschlichen Knotenstruma: die Lösung alter Rätsel? Schweiz med Wochenschr 113:1178-1187-8. Gutekunst R, Smolarek H, Wächter W, Scriba PC (1985) Strumaepidemiologie. IV. Schilddrüsenvolumina bei deutschen und schwedischen Schulkindern. Dtsch med Wochenschr 109:50-54 - 9. Gutekunst R, Smolarek H, Friedrich H-J, Wood WG, Scriba PC (1985) Alimentäre Jodversorgung und Schilddrüsenvolumina in der Bundesrepublik Deutschland und Schweden. Focus MHL (Lübeck) 2:150-158 - 10. Gutekunst R, Smolarek H, Hasenpusch U, Stubbe P, Friedrich H-J, Wood WG, Scriba PC (1986) Goiter epidemiology: Thyroid volume, iodine excretion, thyroglobulin and thyrotropin in Germany and Sweden. Acta Endocr (Kbh): im Druck - 11. Gutekunst R (1986) Strumaepidemiologie in Deutschland. Habilitationsschrift, Medizinische Universität zu Lübeck - 12. Habermann J, Heinze HG, Horn K, Kantlehner R, Marschner I, Neumann J, Scriba PC (1975) Alimentärer Jodmangel in der Bundesrepublik Deutschland. Dtsch med Wochenschr 100: 1937-1945 - 13. Hamburger JI (1981) Should all autonomously functioning thyroid nodules be ablated to prevent the subsequent development of thyrotoxicosis? In: Hamburger JI, Miller JM (eds) Controversies in clinical thyroidology. Springer, New York Heidelberg Berlin, pp 69-104-14. Heberer G, Spelsberg F, Günther B, Kiffner E, Pickardt CR, Scriba PC (1978) Chirurgie der Hyperthyreose. Langenbecks Arch Chir 347:698 - 15. Heidemann PH, Stubbe P, von Reuss K, Schürnbrand P, Larson A, von Petrykowski W (1984) Jodausscheidung und alimentäre Jodversorgung bei Neugeborenen in Jodmangelgebieten der Bundesrepublik. Dtsch med Wochenschr 109:773-778 - 16. Heidemann PH (1986) Jodmangel bei Neugeborenen und Säuglingen. In: Pfannenstiel P et al. (Hrsg) Schilddrüse 1985. Thieme, Stuttgart (in Vorbereitung) - 17. Herrmann J, Emrich D, Kemper F, Köbberling J, Pickardt CR, Stubbe P (1984) Jodexzeß und seine Auswirkungen. Gemeinsame Stellungnahme der Sektion Schilddrüse und der Kommission Hormontoxikologie der Deutschen Gesellschaft für Endokrinologie. Dtsch med Wochenschr 109: 1077-1080 - 18. Homoki J, Birk J, Loos U, Rothenbuchner G, Fazekas ATA, Teller WM (1975) Thyroid function in term newborn infants with congenital goiter. J Pediat 86:753-758 - 19. Horster FA, Klusmann G, Wildmeister W (1975) Der Kropf: Eine endemische Krankheit in der Bundesrepublik? Dtsch med Wochenschr 100:8-9-20. Horster FA, Pfannenstiel P, Hötzel D (1985) Häufigkeit der Jodmangelstruma und ihre Prophylaxe. Dtsch Ärztebl 82:3349-3354 - 21. Joseph K (1981) Potentielle Hyperthyreosen. Früherkennung bei noch eumetabolen Patienten in einem Struma-Endemiegebiet. Dtsch Ärztebl 78:2279-2288 - 22. Kersting M, Gottge M, Wember Th, Weber P, Manz F, Schöch G (1985) Welche Chancen bietet die Verwendung von jodiertem Speisesalz für die Bekämpfung des endemi- 
schen Jodmangels? Ernährungsumschau 32:215 - 23. Klein E, Scriba PC, Pickardt CR (1980) Die blande Struma. In: Oberdisse K, Klein E, Reinwein D (Hrsg) Die Krankheiten der Schilddrüse. Thieme, Stuttgart New York, S 493-529 - 24. Riccabona G (Ed) (1983) 3rd International Thyroid Symposium. Thyroid Cancer. Acta Endocr (Kbh) 102, Suppl 252:1-88 - 25. Scriba PC (1981) Kropfprophylaxe mit jodiertem Speisesalz. Dtsch Ärztebl 78:1677 - 26. Scriba PC, Beckers C, Bürgi H, Escobar Del Rey F, Gembicki M, Koutras DA, Lamberg BA, Langer P, Lazarus JH, Querido A, Thilly C, Vigneri R (1985) Goitre and iodine deficiency in Europe. Report of the Subcommittee for the Study of Endemic Goitre and Iodine Deficiency on the European Thyroid Association. Lancet I: 1289-1293 - 27. Scriba PC (1985) Goitre and iodine deficiency in Europe - a review. Extended Report of the Subcommittee for the Study of Endemic Goitre and Iodine Deficiency of the European Thyroid Association. In: Reinwein D, Scriba PC (eds) Treatment of endemic and sporadic goitre. Schattauer, Stuttgart New York, pp 19-42-28. Stanbury JB, Hetzel BS (1980) Endemic goitre and endemic cretinism. Wiley and Sons, New York - 29. Stewart JC, Vidor GE (1976) Thyrotoxicosis induced by iodine contamination of food - a common unrecognised condition? Brit med J 1:372-375 - 30. Teller WM (1984) Prävention der Neugeborenenstruma durch Jodidbehandlung der Schwangeren. Therapiewoche 34:7093-7096 - 31. von Petrykowski W (1984) Diagnose und Therapie der Struma des Neugeborenen. Therapiewoche 34:7097-7101 - 32. von Reuss K, Wieland OH (1984) Jodmangel, kongenitale Struma und kongenitale Hypothyreose. Ergebnisse des TSH-Screening in Südbayern. Dtsch med Wochenschr 109: 1918-1921 - 33. Weber P, Horster FA, KLett M, Manz F, Schöch G, Kersting M (1986) Verbrauch von jodiertem Speisesalz und Kochsalzumsatz von 42 Familien. In: Pfannenstiel $P$ et al. (Hrsg) Schilddrüse 1985. Thieme, Stuttgart (in Vorbereitung) - 34. Williams I, Ankrett VO, Lazarus JH, Volpe R (1983) Aetiology of hyperthyroidism in Canada and Wales. J Epidemiol Commun Heth 37:245-248 
Adam, O. 600

Aganović, I. 532

Althoff, P.-H. 559

Arnold, K. 366

Barth, C. A. 639

Bax, N. D. S. 497

Beisiegel, U. 383

Beynen, A. C. 666

Billek, G. 588

Bircks, W. 435

Blömer, H. 11

Braun-Falco, O. 325

Brendel, W. 168

Brunner, R. 325

Bünte, H. 175, 371

Deetjen, P. 461

Delvos, U. 120

Demling, L. 346

Distler, A. 307

Dörken, B. 109

Domschke, W. 352

Dresel, H. A. 389

Dunning, A. 397

Efendic, S. 546

Fassbinder, W. 214

Federlin, K. 125

Forth, W. 254

Frühmorgen, P. 362

Fürst, P. 737

Fukagawa, N. 640

Gander, K.-F. 248

Gerok, W. 114

Goebel, F. D. 47, 341

Greten, H. 402

Gries, F. A. 277, 571

Grimble, G. K. 674

Gröbner, W. 455, 492

Grube, U. 224

Gudden, F. 163

Gutekunst, R. 263

Gutensohn, W. 456

Hämmerling, G. J. 109

Häring, R. 114

Hallberg, L. 743

Hartig, W. 224

Hauss, J. 175

Hehlmann, R. 337, 426
Heimpel, H. 425

Helm, E. B. 341

Herzer, P. 53

Herzog, V. 134

Ho, A. D. 85

Hobom, G. 100

Hötzel, D. 263

Holm, E. 685

Holstege, A. 114

Horsthemke, B. 397

Huhn, D. 330

Humphries, S. E. 397

Hunstein, W. 85

Jackson, P. R. 497

Kautz, G. 371

Keller, Ch. 419

Kelley, W. N. 465

Kessling, A. 397

Köhle, K. 452

Kirchner, R. 114

Kleinberger, G. 235

Knorr, M. 289

Köbberling, J. 524

Koschinsky, T. 571

Kramer, H. J. 76

Langhans, P. 356

Leweling, H. 685

Lewis, B. $\quad 383,412$

Lichtlen, P. 435

Lingemann, B. 369

Lison, A.-E. 2

Löffler, W. 484

Louati, M. R. 403

Lüthy, R. 325

Lux, G. 367

Matkowitz, R. 224

Meusel, P. 120

Möller, P. 109

Moldenhauer, G. 109

Momburg, F. 109

Müller-Berghaus, G. 120

Neiss, A. 446

Ostendorf, P. 431

Pausch, J. 114

Pauw, M. 114

Peskar, B. A. 631
Peskar, B. M. 623

Pichlmayr, R. 206

Pongratz, D. E. 508

Possinger, K. 66

Preissner, K. T. 120

Raspe, H.-H. 448

Reiter, S. 512

Riethmüller, G. 106

Rosak, C. 559

Sailer, D. 311

Schattenkirchner, M. 240, 469

Schlierf, G. 292,316

Schöffling, K. 521

Schöllner, D. 235

Schmoz, G. 224

Schreml, W. 439

Schrör, K. 610

Schwandt, P. 595

Schwemmle, K. 192

Scriba, P. C. 263

Searle, J. G. 465

Seeber, S. 202

Seidel, D. 320

Siewert, J. R. 349

Silk, D. B. A. 674

Simmet, Th. 631

Simmonds, H. A. 503

Sinzinger, H. 619

Sirtori, C. R. 403

Skrabalo, Z. 532

Spengel, F. A. 185

Staedt, U. 685

Stähelin, H. B. 23

Standl, E. 579

Steinbach, M. 343

Striebel, J.-P. 685

Stumpe, K. O. 6

Taylor, R. 397

Tschepe, A. 685

Tytgat, G. N. 347

Uhl, W. 685

Ulmer, W. T. 35

Unsöld, E. 153

Usadel, K. H. 61

Utermann, G. 412

Vetter, H. 175

Vetter, W. 289 
Vollmar, J. F. 180

Vranic, M. 546

Walter-Sack, I. 270

Weber, W. 383

Weiner, R. 224
West, C. E. 666

Wilmanns, W. 66

Wilms, K. 89

Wilson, J. W. 465

Winnacker, E. L. 105

Wolfram, G. 247, 587

Woods, H. F. 497
Young, V. R. 640

Ziegler, R. 296

Zöllner, N. XIII, XIX, 1, 99.303

zur Hausen, $H . \quad 114$ 
Adipositastherapie 277

AIDS, Entwicklungen seit $1984325 \mathrm{ff}$.

-, Behandlung opportunistischer

Infektionen 341

-, Definitionen, Klinik und Epidemiologie 325

-, Enzephalopathie 330

-, gesundheitspolitische Maßnahmen 343

-, Klinik und Behandlung des KaposiSarkoms 325

-, Klinik und Therapie maligner Lymphome 330

-, Möglichkeiten der Impfprophylaxe und virusstatischer Therapie 341

Amino Acid Metabolism, with Particular Reference to Nutritional Modulation 640

Amino Acids, in Gastrointestinal Disease 674

Aminosäurenstoffwechsel bei Leberinsuffizienz 685

AMP-Deaminase-Mangel, Myopathie 508

Angiologie, aus Sicht des Chirurgen 180

-, aus Sicht des Internisten 185

Antikörper, monoklonale 109

Antithrombin III, Rolle bei Aktivierung von Protein C 120

Apolipoprotein-Metabolismus, Störungen 403

Apolipoproteins A and C, Mutants of 403

Apolipoprotein E, Mutanten des 412

Arteriosklerose, bei Familiärer Hypercholesterinämie, Pathogenese, neue experimentelle Aspekte 389

Atherogenic Dietary Proteins 666

Bildgebende Systeme, neue 163

Biologie, synthetische 105

Biostatistiker, Darstellung der Prognose aus Sicht des 446

Biosynthese, zellbiologische Grundlagenforschung 134

Bronchialkarzinom, kleinzelliges, intenisive Chemotherapie 439

,-- , therapeutische Strategien

Chemotherapie, intensive, bei kleinzelligem Bronchialkarzinom 439

Diabetes mellitus 311

Diabetes, Typ II 521

-,-, Angiopathien 579

-,-, Differentialtherapie 559

-, -, Epidemiologie of 532

,-- , mellitus, Fettstoffwechsel 571

-, -, New Aspects of Pathogenesis 546
Diagnosis, Use of DNA Deletions and DNA Polymorphisms of LDL 397

Diagnostik, der HTLV-III-Infektion 337

Diätetische Prävention der koronaren Herzkrankheit 292

Dickdarm, entzündliche und neoplastische Krankheiten 362,366

Eicosanoide, klinische Bedeutung 587

- und essentielle Fettsäuren 610

- und Lungenfunktion 631

- und Magen-Darm-Trakt 623

- und periphere Verschlußkrankheit 619

Eisen, Resorption 743

Elektrolythaushalt, Störungen, Indikatoren für neoplastische Prozesse 76

Entscheidungsprozeß, emotionale Situation 452

Enzyme, lipolytische, genetisch bedingte Funktionsstörungen 402

ERCP, bei akuter Pankreatitis 371

Ernährung, parenterale 224, 235

-, Sicherung 248

- , Sonden 224, 235

- und Arzneimittel, aktuelle Probleme 270

- und Hypertonie 289

- und Knochenstoffwechsel 296

- und Krankheit, aktuelle Probleme 247

Familiäre Dyslipoproteinanämie 412

- -, Plasmaaustausch und verwandte Therapieformen bei Schwerer 419

Familiäre Hypercholesterinämie, neue experimentelle Aspekte zur Pathogenese der Arteriosklerose 389

- -, neuere Erkenntnisse zur Pathogenese 383

Fettsäuren, essentielle 610

Fettstoffwechsel, bei Typ II Diabetes mellitus 571

Fettstoffwechselstörungen 316

Fortschritt, medizinischer, Grundlagenforschung 99

Funktionsstörungen, lipolytische Enzyme 402

Gallenwege, therapeutische Grenzgebiete, internistische Aspekte 367

Gallenblase, Stoßwellen-Lithotripsie 168

-, - -, Krankheiten 369

Gastroenterologie, therapeutische Grenzgebiete 346

Gastrointestinal Disease, the Optimum Form of Dietary Nitrogen 674 
Genetik, Analyse des Vererbungsvorgangs 100

-, synthetische Biologie 100

Gentechnologie 105

Gesundheitspolitische Maßnahmen, AIDS 343

Gicht $455 \mathrm{ff}$.

-, klinisches Bild und Diagnose 469

Glomerulonephritis, chronische 2

Grenzgebiet von Innerer Medizin und Chirurgie, Gastroenterologie 346

Grundlagenforschung, Voraussetzung und Fortschritte 99

Herzklappenfehlerkorrektur, internistische Gesichtspunkte 435

-, chirurgische Gesichtspunkte 435

Herzkrankheiten, leiser Beginn 11

-, koronare, diätetische Prävention 292

HGPRT Deficiency 465

HTLV-III-assoziierte Erkrankungen $325 \mathrm{ff}$.

HTLV-III-Infektion, Tests, Infektionsschutz 337

-, gesundheitspolitische Maßnahmen und Aspekte 343

Human Protein, In Vivo, with Particular Reference to Nutritional Modulation 640

Hypercholesterinämie, familiäre, Pathogenese 383

Hyperlipidaemia, Screening and Therapy 412

Hypertension, arterielle 6

Hyperthyreose, Früherkennung schleichender Formen 61

Hypertonie 307

-, endokrine 175

- und Ernährung 289

Hyperurikämie, metabolische Aspekte zur Pathogenese 456

-, Arzneimitteltherapie 492

-, renale Aspekte zur Pathogenese 461

-, Ernährungstherapie 484

Hyperuricaemia, When to Treat 497

Immungenetik, Mensch 106

Immunmangel-Syndrom 325

Impfprophylaxe, bei AIDS 341

Infektionsschutz, bei HTLV-III-Infektion 337

Information des Patienten 448

Jodprophylaxe, Probleme der 263

Kaposi-Sarkom, Klinik und Behandlung bei AIDS 325

Kardiovaskuläres System, Eicosanoide und essentielle Fettsäuren 610

Knochenstoffwechsel, und Ernährung 296

Kollagenosen, Früherkennung und Frühdifferenzierung 53

Kolonkarzinom, modifizierte Nukleoside als Tumormarker 114
Konservierung von Geweben

125

Koronares Risiko 303

- -, Diabetes mellitus 311

- -, Fettstoffwechselstörungen 316

- -, Hypertonie 307

- -, Rauchen 320

Krankheiten, chronische $1 \mathrm{ff}$.

,,-- Beginn, leiser $1 \mathrm{ff}$.

,-- , Frühsymptomatologie $1 \mathrm{ff}$.

,-- , Nosologie der Frühstadien $1 \mathrm{ff}$.

Krebsvirusforschung 114

Kryopräservation und Kultur, Konservierung von Geweben 125

Laser, Möglichkeiten und Grenzen 153

LDL, Use in Diagnosis 397

Leberinsuffizienz, Protein- und Aminosäurenstoffwechsel 685

Leukämie, akute, Vorstadium 85

-, chronisch-myeloische, konventionelle Behandlung 426

-, -, Knochenmarktransplantation 431

Lipidstoffwechsel, angeborene Störungen, Pathogenese $u$. Therapie 383

Lithiasis, 2,8-Dihydroxyadenine, Epidemiology, Pathogenesis and Therapy 503

Lithotripsie, durch Stoßwellen 168

Lungenfunktion, und Eicosanoide 631

Lungenkrankheit, chronische, Frühsymptomatik und Präventionsmöglichkeit 35

Lymphome, maligne, Klinik und Therapie bei AIDS 330

-, -, Vor- und Frühstadien 89

Magen-Darm-Trakt, und Eicosanoide 623

Mineralien, Resorption 743

Molekulargenetik $397 \mathrm{ff}$.

Mutants of Apolipoprotein A and C

Myelodysplasie 85

Myopathie, bei AMP-Deaminase-Mangel 508

Nahrung, Gifte in der 254

Nahrungsproteine, Wahl der $639 \mathrm{ff}$.

,,-- Grundlagen und Diätetik 639

- und Nierenkrankheiten 737

- und Resorption von Mineralien und Spurenelementen 743

-, -, Nutritional Modulation 640

Nephritis, chronische interstitielle 2

Nierenkrankheiten, und Nahrungsprotein 737

Nierentransplantation, internistische Gesichtspunkte 214

Niere, Stoßwellen-Lithotripsie 168

Onkologie 192

Onkologie, internistische, kombiniert internistisch-chirurgische Behandlung maligner Tumoren 202

Organtransplantationen 206 
Pankreas, therapeutische Grenzgebiete, internistische Aspekte 367

-, - -, Krankheiten 369

Pankreatitis, akute, ERCP und endoskopische Sphinkterotomie 371

Parenterale Ernährung 224, 235

Pathogenese, der angeborenen Lipidstoffwechselstörung 383

-, der Hyperurikämie 456, 461

Patienteninformation 448

Peptides, in Gastrointestinal Disease 674

Plasmaaustausch, bei schwerer Familiärer Hypercholesterinämie 419

Plasmalipoproteine, Auswirkung genetisch bedingter Funktionsstörungen lipolytischer Enzyme 402

Polyenfettsäuren, klinische Bedeutung 587

-, Struktur und Zufuhr 588

- und Prostaglandinbiosynthese 600

Protein C, Aktivierung an der Oberfläche kultivierter Endothelzellen 120

Proteins, in Gastrointestinal Disease 674

Proteinstoffwechsel, bei Leberinsuffizienz 685

,--- , infusionstherapeutische und diätetische Folgerungen 685

Psychoorganische Veränderungen 23

Purinstoffwechsel, Störungen 455

Pyrimidinstoffwechsel, Störungen 455

Rheumatologie 235, 240

Risiko, koronares 303

Serumlipoproteine 595

Sondenernährung 224, 235

Speiseröhre, Krankheiten, therapeutische Grenzgebiete 347,349

Sphinkterotomie, endoskopische, bei akuter Pankreatitis 371

Spurenelemente, Resorption 743

Stoßwellen-Lithotripsie, in Niere und Gallenblase 168

Syndrome, paraneoplastische 66

Systeme, neu bildgebende 163
Therapeutische Grenzgebiete, Gastroenterologie 346

- -, Dickdarm, entzündliche und neoplastische Krankheiten 362, 366

- -, internistische Aspekte der Krankheiten der Gallenwege und des Pankreas 367

- -, Krankheiten der Speiseröhre 347, 349

- -, Ulkuskrankheit, Standpunkt des Chirurgen 356

- -, -, Standpunkt des Internisten 352

Therapie, virustatische, der HTLV-III/LAVInfektion 341

-, der angeborenen Lipidstoffwechselstörungen 383

-, Alternativen zwischen palliativer und kurativer 425

Thyreoglobulintransport, zellbiologische Grundlagenforschung 134

Tumordiagnostik, monoklonale Antikörper 109

Tumore, maligne 66

,-- , Früherkennung 66

,,-- kombinierte internistisch-chirurgische Behandlung 202

Tumormarker, Kolonkarzinom, modifizierte Nukleoside 114

Tumortherapie, monoklonale Antikörper 109

Urinausscheidung, erhöhte, Wertigkeit 114

Verschlußkrankheit, periphere, und Eicosanoide 619

Virus, HTLV-III/LAV 341

Wasserhaushalt, Störungen, Indikatoren für neoplastische Prozesse 76

Wegener'sche Granulomatose, Frühstadien und Verlauf 47

Zuckerkrankheit, Klassifikation und Vererblichkeit 524 